\title{
High Frequency Modulation Method for Measuring of Birefringence
}

\author{
Š. Kunc, M. Šulc \\ Technical University of Liberec, Physics Department, 46117 Liberec, Studentská 2, Czech Republic
}

\begin{abstract}
A method of optical birefringence measurement is presented. It uses an electro-optic modulator for the high frequency modulation of polarization of the laser beam. The developed optical apparatus exhibits high sensitivity. It is able to measure very small birefringence of samples down to $10^{-3} \mathrm{rad}$. The accuracy and sensitivity of the method was checked by measurement of calibrated Soleil - Babinet compensator. Method can be also used for online and accurate measurement of an optical components birefringence. This application was developed with the aim to measure Cotton-Mouton effect in air and nitrogen.
\end{abstract}

\section{Introduction}

Optical birefringence is a well know phenomenon for many decades. It is observed in optical materials used in industrial applications, such as wave plates, photomask windows and liquid crystals. Consequently, it is necessary to apply a precise degree of control to the high precision optical components and instruments employed in modern semiconductor, electro-optic and related industries. Optical retardation can be also induced by external forces such as stress, thermal gradient, electric and magnetic field. It is often unwanted phenomenon which is necessary to check in fabrication process. Through birefringence, we can consider a wide range of physical and biophysical properties. Even we can verify through birefringence basic physical theories.

Birefringence has been measured by a variety of techniques. We will mention only some of them. Phase retardation, and thereby birefringence, may be determined by a measurement of the spectral intensity of chromatic fringes in channelled spectrum [1]. A compensator or a quarter-wave plate can be used with polarimeter. The retardation is determined from the phase compensation or the analyzer rotation; that is required to give intensity; null [2]. Such methods are relatively simple but they have disadvantages that often include manual adjustment and scale reading, a nonlinear relation between birefringence and light intensity, and only modest sensitivity and precision. Static methods were overcome by modulation techniques. The modulation can be introduced by rotating retardation plate [3], a photoelastic modulator [4], an analyzer rotating or by Faraday rotator in combination whit quarter wave plate [5]. We will be able to find others methods of modulation. Compensation of linear working point of electro-optic modulator can be used to determinate retardation of the sample [6].

In this method we have used high frequency electrooptic modulator against elasto-optic modulator which is more stable but does not provide high modulation frequency. Elasto-optic modulators work mostly at $50 \mathrm{KHz}$ or $100 \mathrm{Khz}$ (resonant frequency) and typically are not frequency-tunable. On the other hand Electro-optic modulators are able to modulate up to $\mathrm{GHz}$ frequency and are tunable from $0 \mathrm{~Hz}$. High modulation frequency provides better signal to noise ratio because of its $1 / \mathrm{f}$ dependence. The method described in this article is able to measure very small retardation as in our case CottonMouton effect in air and nitrogen. In this paper we present optical set-up and procedures of testing measurement with Soleil-Babinet compensator. Possible errors and their solution associated with the use of electro-optic modulator are briefly discussed in the conclusion.

\section{Apparatus}

Figure 1 shows the set-up of the optical apparatus. A laser beam is linearly polarized by a polarizer prism (Glan-Thompson polarizer). The beam then goes through the electro-optical modulator; an optical device that gives ellipticity to the beam (phase shift between two principal polarizations), relative to the applied electric signal. The beam then propagates trough birefringence sample (birefringence material, induced birefringence) where the light acquires an ellipticity from optical anisotropy. The polarization of the beam is finally analyzed by a polarizer prism (analyzer), and light intensity is detected by photodiode. The electro-optic modulator converses linearly polarized light into light oscillating between left and right ellipticity at $49.4 \mathrm{kHz}$ (maximum frequency is

This is an Open Access article distributed under the terms of the Creative Commons Attribution License 2.0, which permits unrestricted use, distribution, and reproduction in any medium, provided the original work is properly cited. 
limited by second harmonic signal for $100 \mathrm{kHz}$ Lock-in amplifier, modulator can operate up to $50 \mathrm{Mhz}$ ). Electrooptic modulator does not work in his linear point but oscillates around minimum valley "point of second harmonic", see figure 2. The set of possible configurations of polarizing elements was investigated. The best orientation of each successive component in the set-up is at 45 degrees rotation to its previous element [4].

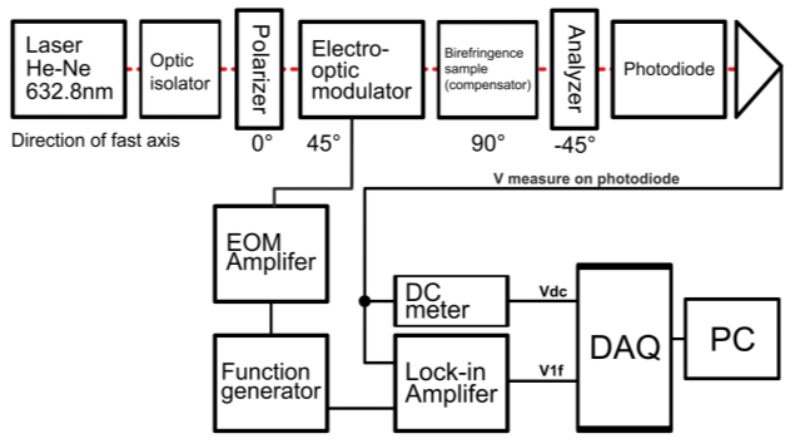

Fig. 1. Optical apparatus with the orientation of the optical components.

Optical components of our apparatus were chosen for $632.8 \mathrm{~nm}$ laser and for low level of intensity $1 \mathrm{~mW}$. Glan-Thompson prisms (CVI Melles Griot) were used for polarizating and analyzing of light. They provides excellent extinction ratio 1:100000. The high frequency electro-optic modulator is made by Quantum Technology Ltd., and provides tunable modulation from $0 \mathrm{~Hz}$ to $50 \mathrm{MHz}$. Modulator is realized by four ADP crystals for better thermal compensation and is designed as travel wave modulator to achieve $\mathrm{MHz}$ modulation frequency. Photodiode is Hammatsu model C6236, using optical fiber input. AC modulation signal was build up by wave function generator and measured system response was analyzed by $100 \mathrm{kHz}$ dual phase Lock-in amplifier Stanford Research 830 DSP and DC voltage on photodiode was measured by Orbit Meret DC meter.

A Jones calculus computation [7] gives the light intensity $I$ transmitted by the analyzer in terms of the intensity $I_{0}$ transmitted by the polarizer. For our assembly is the result of,

$$
I=I_{0} / 2(1+\sin \delta \sin T)
$$

Where $T$ is the phase shift induced by the modulator, and the phase retardation $\delta$ of the sample:

$$
\begin{gathered}
\delta=2 \pi \Delta n(L / \lambda) \\
T=T_{0} \sin \omega t \\
T_{0}=\left(V_{\mathrm{m}} / V \pi\right) \pi
\end{gathered}
$$

where $\Delta n$ is sample birefringence, $L$ is sample thickness or length of propagation in anisotropic media, $\lambda$ the wavelength of used laser in vacuum $(632.8 \mathrm{~nm}), T_{0}$ the amplitude of the modulator retardation (amplitude modulation index or depth of modulation) that is proportional to the amplitude of applied oscillating voltage $V_{\mathrm{m}}$ and inverse to half wave voltage $V \pi(125.8 \mathrm{~V}$ DC) of the electro-optic modulator. The measured values of intensity as a function of applied DC voltage are presented at Figure 2. According to equation (1) thus any birefringence in the sample produced amplitude of the modulation of the intensity detected by the photodiode. Parasitic birefringence, coming from optical components, can be compensated by the Soleil-Babinet compensator.

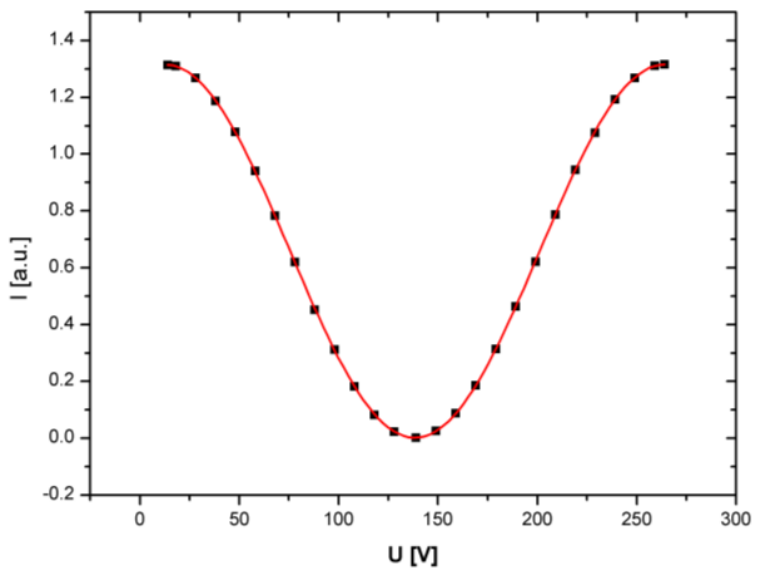

Fig. 2. A modulator DC Bias plot for crossed polarizer and analyzer.

It is useful to maximize the first harmonic component of the modulated signal to achieve the best sensitivity of apparatus. Detected signal of the modulated intensity has a harmonic content [8] described by

$$
\sin \left(T_{0} \sin \omega t\right)=2 \sum_{\mathrm{m}=\text { odd }} J_{\mathrm{m}}\left(T_{0}\right) \sin m \omega t
$$

The Bessel function $J_{1}\left(T_{0}\right)$ has maximum for $T_{0}=105^{\circ}$. Our modulator is able to produce maximum $T_{0}=72^{\circ}$ of modulation depth [9], see Figure 3 . We measured calibration plot of modulator for different values of modulation depth. It fits with theoretical data of Bessel function well. Better agreement has been reached for a greater depth of modulation.

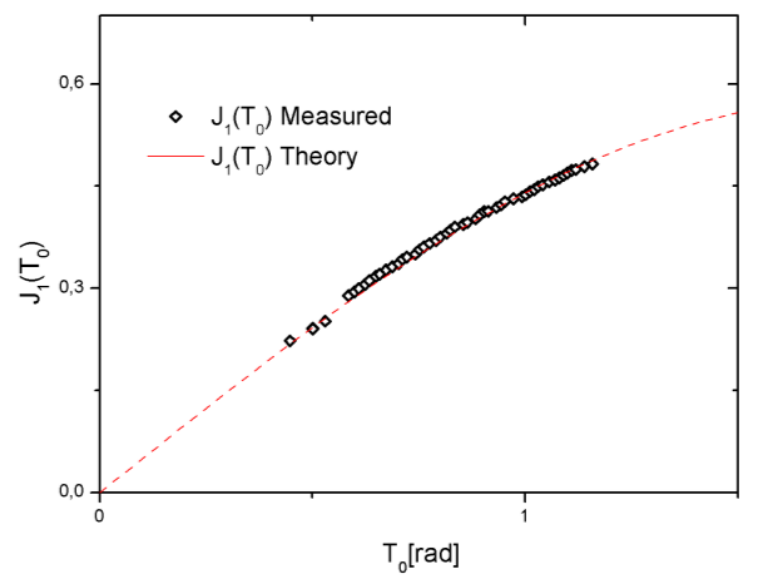

Fig. 3. A modulator calibration plot. 
If we were able to achieve a modulation depth across the half-wave voltage $\left(V_{\mathrm{m}}=V \pi\right)$, using the saw-tooth signal build by function generator, analysis of the AC signal from photodiode would become much simpler [10].

\section{Procedures}

The electro-optic modulator may be aligned between crossed polarizer and analyzer by maximization of the second harmonic $(98.8 \mathrm{kHz})$ signal or by minimization of the first harmonic signal. Propper orientation of the analyzer is then realized by turning it to null second harmonic signal, in the same time we have to check the bias of electro-optic modulator to precisely set the operating point to minimum or zero phase. Combination of precise orientation and controlling bias will set the modulator for correct measurement. If the birefringence sample is then inserted, it may be oriented by maximization the fundamental first harmonic (49.4 $\mathrm{kHz}$ ) signal [10] and again we have to check operation point via bias voltage.

For small retardation it is best to use the phase sensitive detection provided by dual phase DSP Lock-in amplifier to measure the first harmonic of AC signal, and thus determine $\sin \delta$. If a retardation of the sample is very small we can well approximate $\sin \delta$ as $\delta$. We can simply calculate $\delta$ as:

$$
\delta=\arcsin \left(\sqrt{ } 2 V_{1 \mathrm{f}} / V_{\mathrm{dc}} 2 J_{1}\left(T_{0}\right)\right)
$$

where, $V_{1 \mathrm{f}}$ is rms AC output intensity (R value) of DSP Lock-in amplifier for first harmonic component of the signal and $V_{\mathrm{dc}}$ is DC intensity on photodiode. Measured data were collected using NI USB data acquisition system and subsequently evaluated in computer.

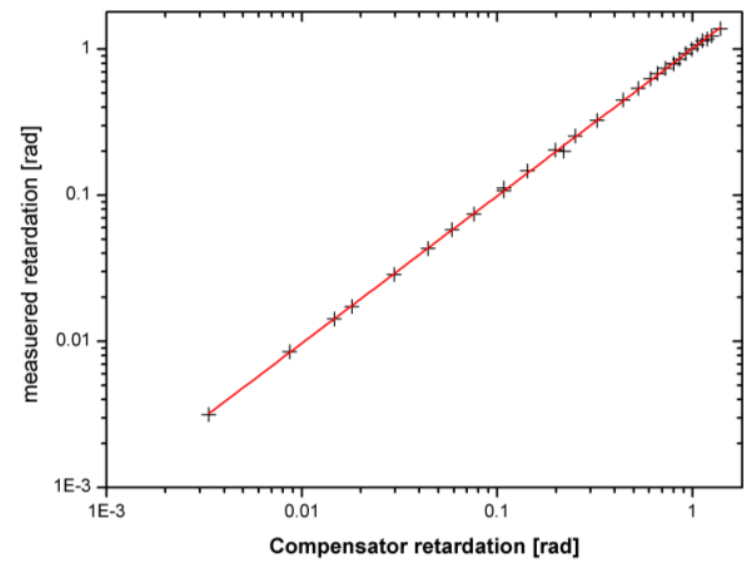

Fig. 4. Confidence plot for compensator retardation and measured retardation.

\section{Results}

Figure 4 shows the results of the measurement made with Soleil-Babinet compensator as the variable birefringence sample. Compensator is able to provide full wave retardance adjustment. Digital reed out resolution is $0.001 \mathrm{~mm}$ and corresponding repeatability value at
$633 \mathrm{~nm}$ is 0.001 waves. Because the sine function is symmetric, we are able to measure only in region from zero to ninety degrees, where around maxima gradually loses resolution. Method was developed for precise measuring very small birefringence and therefore this problem with lack of resolution or intensity of AC signal can be neglected. Confidence plot fig. 4 represents good agreement between measured retardation and values, represented by the compensator. Pearson's $r$ value of linearity confidence is 0.9999 , slope value of linear fit is $1.0086 \pm 0.0027$, intercept is $0.0040 \pm 0.0026$.

\section{Conclusion}

We have reported a technique for measuring the birefringence. The method is experimentally simple but is sensitive to accurate orientation of the optical elements and good linear response of electronic components. Possible errors from misorientation can be reduced by precise adjustment or by servo control. We have observed small instability of electro-optic modulator, which can be compensated by feedback loop of bias voltage or by precise control of temperature [11]. Feedback loop can be set for example to monitor the minimum of second harmonic in AC signal. Feedback plays very important role in long-term measurements. We have also observed very slow changing spurious signals at fundamental frequency, which can influence long term measurements. Problem with spurious signal is possible to reduce by monitoring reference signal placed before birefringence sample. Reference signal can be digitally subtracted during processing result. The apparatus is sensitive enough to measure Cotton-Mouton effect in air and nitrogen in $14.3 \mathrm{~m}$ long and 9T strength LHC dipole magnet. This is expected to give retardation of 0.0035 rad. for nitrogen, $1 \mathrm{~atm}$. $632.8 \mathrm{~nm}$ wave length.

\section{Acknowledgments}

This work was supported by the SGS Grant " Accurate measurement of birefringence using highfrequency electro-optic modulation" of Technical University of Liberec and partly supported by the Grant Agency of the Czech Republic 203/11/1546.

\section{References}

1. T. E. Walsh, J. Opt. Soc. Am 62, 81 (1972)

2. T. B. Brown, The Taylor Manual, Ed. AddisonWesley, Reading, Mass., (1959)

3. J. Badoz, J. Phys. Radium 17, 143 A (1956)

4. F.A. Modine, R. W. Major, E. Sonder, Applied optics 14, 3 (1975)

5. S. Carussoto, E. Polacco, E. Iacopini, G. Stefanini, E. Zavattini, Optic communications 42, 2 (1982)

6. BE. Benkelfat, EH. Horache, Q. Zou, B. Vinouze, Optics Communications 221, 271-278 (2003)

7. R. C. Jones, J. Opt. Soc. Am. 38, 671 (1948) 
8. H. J. Bartsch, Matematicke vzorce. Mlada fronta, Praha (2002)

9. J. R. Mackey, K. K. Das, S. L. Anna, G. H. McKinley, Meas. Sci. Technol. 10, 946-955 (1999)

10. S. E. Lee, J. F. Lin, Y. L. Lo, Optics and Laser in Engineering 43, 704-720 (2005)

11. D. T. Bui, L. D. Nguyen, B. Journet, REV Journal on Electronics and Comunications, 1, 1 (2011) 